\title{
Zumbido: possível associação com alterações cervicais em idosos
}

\section{Tinnitus: probable association with the elderly's cervical alterations}

\author{
Michelle Damasceno Moreira', Luciana Lozza de Moraes Marchiori², Viviane de Souza Pinho Costa ${ }^{3}$, Erick Costa Damasceno4, \\ Paula Carolina Dias Gibrin ${ }^{5}$.
}

1) Especialista, Mestranda em Ciências da Reabilitação UNOPAR. Fisioterapeuta concursada pelo Programa Saúde da Família.
2) Doutora em Medicina e Ciências da Saúde. Docente do Mestrado em Ciências da Reabilitação UNOPAR.
3) Doutora em Enfermagem, Escola de Enfermagem de Ribeirão Preto, USP. Professor, Universidade Norte do Paraná, PR.
4) Bacharel em Estatística - Universidade de Brasília, 2008. Sócio-Diretor da Odds\&Actions e Conselheiro do Conselho Regional de Estatística $1^{\text {an Região. }}$
5) Especialista em Audiologia, mestranda em Ciências da Reabilitação UNOPAR. Fonoaudióloga do Cismepar.

Instituição: Universidade do Norte do Paraná - UNOPAR.

Londrina / PR - Brasil.

Endereço para correspondência: Michelle Damasceno Moreira - Rua Augusto Guerino, 195 Apto 35 - Bairro: Portal de Versailhes 1 - Londrina / PR - Brasil - CEP: 86057-240 - Telefone: (+55 43) 3178-0336/3371-7990/9161-5577 - E-mail: micmoreira@yahoo.com.br

Artigo recebido em 6 de Abril de 2011. Artigo aprovado em 23 de Maio de 2011.

\section{RESUMO}

Introdução: O zumbido é um sintoma prevalente e de alto impacto na qualidade de vida do paciente idoso. A presença de cervicalgia e alterações na coluna cervical são comuns em pacientes com queixa de zumbido.

Objetivo: Avaliar a prevalência do zumbido e cervicalgia em um grupo de idosos e verificar a possível associação entre zumbido, cervicalgia e restrição de amplitude de movimento cervical.

Método: Estudo transversal retrospectivo com avaliação da amplitude de movimento cervical através de goniometria e do zumbido e da cervicalgia através da aplicação de questionário padronizado.

Resultados: A amostra foi constituída por 147 indivíduos, com idade média de 69,22 anos sendo 61,90\% mulheres. Desses indivíduos, 42,85\% apresentaram queixa de zumbido 51\% dos indivíduos relataram queixa de dor cervical. Não houve associação entre zumbido e dor cervical e nem associação entre zumbido e restrição de amplitude de movimento cervical.

Conclusão: Embora não tenha sido constatada associação entre zumbido e dor cervical e entre zumbido e restrição de amplitude de movimento, observou-se grande prevalência de queixa de zumbido, de cervicalgia e de diminuição na amplitude de movimento cervical na população de idosos. Os resultados da presente pesquisa, por meio da constatação desta grande prevalência do zumbido em toda a população de estudo, servirão de base a uma integração entre profissionais da área de saúde envolvidos com tais alterações.

Palavras-chave: zumbido, cervicalgia, coluna vertebral, idoso.

\section{SUMMARY}

Introduction: Tinnitus is a prevailing symptom that highly jeopardizes the elderly patient's quality of life. Neck pain and cervical column alterations are frequent in patients complaining about tinnitus.

Objective: Evaluate the prevalence of both tinnitus and neck pain on an elderly group, and verify the likely association between tinnitus, neck pain and the constraint to make wide cervical movements.

Methods: Retrospective transversal study, evaluating both the width of cervical movement by way of a goniometry and the tinnitus and neck pain by requesting a standard questionnaire to be filled out.

Results: Sample was comprised of 147 individuals aged between 69.22, 61.90\% of whom were female. Among these individuals, $42.85 \%$ showed a buzz complaint and $51 \%$ of these individuals claimed to have a neck pain. Neither was association found between tinnitus and neck pain nor it was between tinnitus and the width constraint of cervical movements. Conclusion: Despite not finding an association between tinnitus and neck pain or between tinnitus and the width constraint of cervical movements, there was a significant prevalence of tinnitus, neck pain and a reduction in the width of cervical movements on the elderly people. The results hereof, by finding this important prevalence of tinnitus in all the individuals of this study, will be the basis to integrate health professionals engaged in such alterations.

Keywords: tinnitus, neck pain, vertebral column, elderly. 


\section{INTRODUÇÃO}

O aumento da idade é diretamente proporcional à presença de múltiplos sintomas auditivos, tais como, vertigem, presbiacusia e zumbido (1). Além disso, a prevalência de degeneração na articulação cervical e cervicalgia em indivíduos acima de 50 anos é alta, com a tendência de aumentar a severidade com a idade (2).

O zumbido é um sintoma definido como a percepção de um som nos ouvidos ou na cabeça sem que haja produção do som por uma fonte externa. Afeta aproximadamente $15 \%$ da população mundial e esta prevalência aumenta para 33\% entre os indivíduos com mais de 60 anos de idade (3). O zumbidoé um sintoma que frequentemente acompanha a presbiacusia e costuma ser mais perturbador que a própria perda auditiva (4).

Atualmente, sabe-se que o zumbido surge como resultado da interação dinâmica de vários centros do sistema nervoso e do sistema límbico e que as alterações e ou lesões na cóclea são as precursoras deste processo, causando desequilíbrio nas vias inferiores do sistema auditivo, resultando em atividade neuronal anormal, mais adiante realçada pelo sistema nervoso central e, finalmente, percebida como zumbido (3). Além disso, aferências a partir da porção cervical com projeções para o núcleo coclear, indicam uma influência do reflexo da coluna cervical a este centro de audição (5).

Entre as inúmeras etiologias do zumbido estão as doenças otológicas, metabólicas, cardiovasculares, patologias da coluna cervical, odontológicas, neurológicas, psiquiátricas e outras relacionadas com ingestão de drogas, cafeína, álcool e tabagismo (6,7). Eainda, a diminuição da acuidade auditiva, exposição à ruídos, lesões de cabeça e cervical,fatores de estilo de vida e estado mental (8).

A presença de queixa de cervicalgia e cefaleia é comum em pacientes com zumbido e ainda, verifica-se que estes pacientes podem ter o seu sintoma influenciado pela presença de pontos-gatilho miofasciais nos músculos posturais da região cervical, na cintura escapular e musculatura mastigatória, onde provocam dor espontânea ou ao movimento (9). Pacientes com dor crônica, particularmente com dor miofascial em região cervical ou doença degenerativa da coluna cervical, a incidência de zumbido e hiperacusia é mais elevada do que seria previsível da população em geral (10).

O objetivo deste estudo foi investigar a prevalência do zumbido e alterações cervicais em pacientes idosos do projeto EELO (Estudo do Envelhecimento e Longevidade) e verificar suas possíveis associações.

\section{MÉTODO}

Foi realizado um estudo transversal em que os critérios foramà inclusão de pessoas idosas com idade igual ou superior a 60 anos, de ambos os sexos, com vida independente, que estavam classificados nos níveis 3 e 4 do Status Funcional proposto por SPIRDuso (11), e que aceitaram participar voluntariamente do estudo.

Foram alocados os primeiros 147 indivíduos do projeto EELO (estudo sobre envelhecimento elongevidade) no município de Londrina. A amostragem foi definida de forma aleatória estratificada, levando-se em consideração as cinco regiões do município.

Neste estudo, foram mensurados os movimentos ativos de flexão, extensão, flexão lateral de neutro para a direita e para a esquerda, rotação em neutro para a direita e para a esquerda da coluna cervical para avaliar se existe a diminuição de amplitude de movimento na população estudada através da goniometria utilizando um goniômetro universal (Carci, Indústria e Comércio de Aparelhos Cirúrgicose Ortopédicos Ltda, Brasil) com escala de medida de dois em dois graus. Para mensuração da ADM cervical através da goniometria foi considerado o sistema de mensuração desenvolvido por KapandjI (12) e MARQues (13), sendo de flexão cervical de 0 a $65^{\circ}$, extensão cervical de $0-50^{\circ}$, flexão lateral para a direita e esquerda de 0 a $40^{\circ}$ e rotação cervical de 0 a $55^{\circ}$.A presença de cervicalgia e o zumbido foram verificados através da aplicação de questionário padronizado, utilizado durante as coletas do projeto EELO.

Esta pesquisa foi aprovada pelo comitê de ética em pesquisa da UNOPAR, protocolo número 0070/09.

Foi realizado estudo com os testes Qui-quadrado e Risco Relativo para verificar as possíveis associações entre a cervicalgia e os indivíduos que apresentaram e os que não apresentaram zumbido, além de mensuração de amplitude de movimento de cervical, para verificar se havia restrição na mobilidade articular e a possível associação com o zumbido.

Foram considerados para as análises univariadas $p<0,01$ e para inclusão no modelo final para o teste Quiquadrado e para o Risco Relativo o valor de $p<0,05$, ambos com intervalo com $95 \%$ de confiança. O programa estatístico utilizado foi o SAS 9.1.3

\section{RESULTADOS}

A amostra foi constituída por 147 indivíduos em idade entre 60 e 95 anos e com média de idade entre 69,22 
Tabela I. Zumbido por Dor Cervical.

\begin{tabular}{llccc}
\hline Zumbido & Dor Cervical & & & \\
\hline Frequência & & Não & Sim & Total \\
& Não & 45 & 39 & 84 \\
& Sim & 27 & 36 & 63 \\
\hline Total & & 72 & 147 & 75 \\
\hline
\end{tabular}

Qui-quadrado $P=0,1984$

Tabela 2. Zumbido versus Limitação de Movimento da Cervical.

\begin{tabular}{|c|c|c|c|c|c|c|c|}
\hline Variável & Média & $\begin{array}{l}\text { Limite Inferior } \\
\text { do IC }(95 \%, \mu)\end{array}$ & $\begin{array}{l}\text { Limite Superior } \\
\text { do IC }(95 \%, \mu)\end{array}$ & Desvio padrão & $\mathrm{N}$ & Mínimo & Máximo \\
\hline Lim.flex_ce & | 83.642.857 & 164.188 .378 & 203.097 .336 & 116.4 & 140 & 0 & 500.000 .000 \\
\hline Lim_ext_cerv & 69.857 .143 & 56.859 .292 & 82.854 .994 & 26 & 140 & 0 & 300.000 .000 \\
\hline Lim_rot_cervd & 100.647 .482 & 87.353 .766 & || $3.94 \mid .198$ & 64.816 & 139 & 0 & 350.000 .000 \\
\hline Lim_rotcerv_e & $83.2 \mid 4.286$ & $72.304 .87 \mid$ & 94.123 .700 & 65.285 .955 & 140 & 0 & 250.000 .000 \\
\hline LIm flex lat $\mathrm{d}$ & $|25.899 .28|$ & ||$|.92| .55 \mid$ & 139.877 .010 & 83 & 139 & 0 & 300.000 .000 \\
\hline Lim_flex_lat_e & $|32.928 .57|$ & | 19.043.700 & $|46.8| 3.443$ & 83.092 .186 & 140 & 0 & 300.000 .000 \\
\hline
\end{tabular}

Legenda: Lim.flex.cerv :limitação em flexão de cervical; Lim ext cerv: limitação em extensão cervical; Lim rot cerv d: Limitação de rotação cervical para a direita; Lim flex cerv e: limitação de rotação cervical para a esquerda; Lim flex lat d: limitação em flexão lateral para a direita; limitação em flexão lateral para a esquerda.

Tabela 3.

\begin{tabular}{lllccc}
\hline & & \multicolumn{2}{c}{ Teste-T } & & \\
Variável & Método & Variância & Graus deliberdade(GL) & Valordot & $P>|\mathrm{t}|$ \\
\hline $\lim _{\text {_flex_cerv }}$ & Pooled & Equal & 138 & 0.90 & 0.3700 \\
lim_flex_cerv & Satterthwaite & Unequal & 129 & 0.90 & $0.37 \mid 8$ \\
lim_ext_cerv & Pooled & Equal & 138 & 0.22 & 0.8258 \\
lim_ext_cerv & Satterthwaite & Unequal & 127 & 0.22 & 0.8270 \\
lim_rot_cer_d & Pooled & Equal & 137 & 0.19 & 0.8470 \\
lim_rot_cer_dd & Satterthwaite & Unequal & 136 & 0.20 & 0.8447 \\
lim_rot_cer_e & Pooled & Equal & 138 & 0.23 & 0.8170 \\
lim_rot_cer_ee & Satterthwaite & Unequal & 130 & 0.23 & 0.8172 \\
lim_flex_lat_d & Pooled & Equal & 137 & 1.06 & 0.2927 \\
lim_flex_lat_d & Satterthwaite & Unequal & 131 & 1.06 & 0.2920 \\
lim_flex_lat_e & Pooled & Equal & 138 & 0.11 & 0.9163 \\
lim_flex_lat_e & Satterthwaite & Unequal & 135 & 0.11 & 0.9155 \\
\hline
\end{tabular}

Legenda: Lim.flex.cerv :limitação em flexão de cervical; Lim ext cerv: limitação em extensão cervical; Lim rot cerv d: Limitação de rotação cervical para a direita; Lim flex cerv e: limitação de rotação cervical para a esquerda; Lim flex lat d: limitação em flexão lateral para a direita; limitação em flexão lateral para a esquerda.

anos sendo 61,90\% mulheres (91) e 38,10\% homens (56). Desses indivíduos, 42,85\% (63) apresentaram queixa de zumbido 36,50\% homens (23) e 63,50\% mulheres (40), dos que apresentaram zumbido (63), 61,90\% apresentaram zumbido bilateralmente, 26,99 na orelha direita e $11,11 \%$ na orelha esquerda. E ainda, $51 \%$ dos indivíduos relataram queixa de dor cervical, sendo que destes 70,7\% eram do sexo feminino. Ao realizar o teste Qui-quadrado e para o Risco não houve evidências para acreditar que exista associação entre zumbido e dor cervical já que o $p=0,1984$ $(p>0,05)$ (Tabela 1)

Os idosos obtiveram uma limitação em todas as amplitudes de movimentos, conforme a Tabela 2. Quando comparado à presença de zumbido com a amplitude de movimento da cervical, não houve associação entre a limitação de movimento da cervical entre pessoas com e sem zumbido, $p>0,05$. (Tabela 3)

Se desconsiderar o fator zumbido, há diferença na amostra entre o tamanho das limitações de rotação cervical de acordo com o lado. Nesse caso, a restrição de amplitude de movimento é mais limitada para o lado direito do que para o lado esquerdo $p=0.0324$ Tabela 4 .

Não houve associação entre o lado afetado pelo zumbido e o lado da restrição de amplitude de movimento, nos indivíduos com queixa de zumbido $p>0,05$. 
Tabela 4.

\begin{tabular}{|c|c|c|c|c|c|c|c|c|c|c|c|}
\hline \multicolumn{12}{|c|}{ Estatística } \\
\hline Variável & Lado & $N$ & $\begin{array}{c}\text { Limite } \\
\text { Inferior } \\
\text { do IC } \\
(95 \%, \mu)\end{array}$ & Média & $\begin{array}{c}\text { Limite } \\
\text { Superior } \\
\text { do IC } \\
(95 \%, \mu)\end{array}$ & $\begin{array}{c}\text { Limite } \\
\text { inferior } \\
\text { IC } \\
\text { Desvio- } \\
\text { Padrão }\end{array}$ & $\begin{array}{l}\text { Desvio- } \\
\text { Padrão }\end{array}$ & $\begin{array}{l}\text { Limite } \\
\text { Superior } \\
\text { Desvio- } \\
\text { Padrão }\end{array}$ & $\begin{array}{c}\text { Erro- } \\
\text { padrão }\end{array}$ & Mínimo & Máximo \\
\hline limite_rot_cerv & DIREITO & 139 & 8.7354 & 10.065 & 11.394 & 7.0915 & 7.9265 & 8.9862 & 0.6723 & 0 & 35 \\
\hline limite_rot_cerv & ESQUERDO & 140 & 7.2305 & 8.3214 & 9.4124 & 5.843 & 6.5286 & 7.3978 & 0.5518 & 0 & 25 \\
\hline limite_rot_cerv & Diff $(1-2)$ & & 0.0324 & 1.7433 & 3.4543 & 6.7013 & 7.2587 & 7.9181 & 0.8691 & & \\
\hline limite_flex_lat & DIREITO & 139 & 11.192 & 12.59 & | 3.988 & 7.4563 & 8.3343 & 9.4485 & 0.7069 & 0 & 30 \\
\hline limite_flex_lat & ESQUERDO & 140 & | I.904 & 13.293 & | $4.68 \mid$ & 7.4367 & 8.3092 & 9.4155 & 0.7023 & 0 & 30 \\
\hline limite_flex_lat & Diff $(1-2)$ & & -2.664 & -0.703 & I.2586 & 7.6827 & 8.3217 & 9.0776 & 0.9964 & & \\
\hline
\end{tabular}

Legenda: Lim.flex.cerv :limitação em flexão de cervical; Lim ext cerv: limitação em extensão cervical; Lim rot cerv d: Limitação de rotação cervical para a direita; Lim flex cerv e: limitação de rotação cervical para a esquerda; Lim flex lat d: limitação em flexão lateral para a direita; limitação em flexão lateral para a esquerda.

\section{DISCUSSÃO}

A prevalência de zumbido nesta população foi de $40 \%$. Em um estudo no Japão a prevalência de zumbido foi de 18,6\% em indivíduos acima de 65 anos, na Austrália a prevalência foi de $32,7 \%$ acima de 60 anos e na Nigéria $41,9 \%$ em indivíduos acima de 80 anos $(7,8)$. O zumbido pode afetar direta ou indiretamente o indivíduo nas suas atividades profissionais e de lazer, interferir em relacionamentos familiares e sociais podendo levar, em casos extremos, até mesmo ao suicídio. Estudos têm demonstrado que o zumbido pode reduzir qualidade de vida e diminuir a sensação de bem-estar em populações de idosos (14).

Em estudo brasileiro, de 406 pacientes avaliados no período de seis meses, $58 \%$ apresentaram queixa de zumbido, sendo que, destes, $68 \%$ eram do sexo feminino e $32 \%$ eram do sexo masculino o que é compatível estes resultados, em que dos idosos que apresentavam zumbidos $63,50 \%$ eram mulheres (15).

Os estudos são controversos no que diz respeito à influência do sexo na prevalência do zumbido. Apesar de alguns mostrarem discreto aumento da prevalência no sexo feminino, outros sugerem maior prevalência no sexo masculino, mas raramente se alcança significância estatística. A possível justificativa para a maior prevalência no sexo masculino seria o fato de os homens estarem mais expostos ao ruído ocupacional. Por outro lado, as mulheres apresentam maior disponibilidade de tempo para procurar auxílio médico, o que explicaria o achado de maior prevalência no sexo feminino. Neste estudo também houve maior predomínio de pacientes do sexo feminino (3).

Foi verificado que $51 \%$ da população do estudo apresentava queixa de cervicalgia, equivalente a estudos em que a prevalência de dor cervical na população adulta pode variar de 6 a 50\% (16). Tais sintomas são decorrentes de vários processos degenerativos, traumas diretos, ou em razão de microtraumas cumulativos do estresse postural. A redução de massa muscular entre 50-80 anos de idade, provavelmente é resultado do processo de envelhecimento do sistema neuromuscular combinado com a diminuição do nível de atividade física (2).

Pesquisa desenvolvida por um grupo de pesquisa em zumbido mostrou que este sintoma também apresenta uma forte associação com a presença de pontos gatilhos miofaciais nas regiões de cabeça, pescoçoe cintura escapular (9). Além disso, foi demonstrado que em pacientes com zumbido, 75\% dos pacientes apresentavam influência na intensidade do zumbido dependente da movimentação da cabeça e do pescoço (18). Embora a alta prevalência da cervicalgia e zumbido em nosso estudo, não houve associação entre essas duas queixas.

Os idosos deste estudo apresentaram redução de amplitude de movimento em todos os graus de movimento, sendo o maior em flexão cervical. Porém não houve associação entre o zumbido e a redução de amplitude de movimento. A amplitude diminui com a idade de forma consistente em praticamente todos os estudos e aparentemente não há influências intrínsecas que consigam diminuir essa progressão. A redução de amplitude de movimento cervical é multifatorial. Deve-se levar em consideração, os processos degenerativos, sejam eles discais, ósseos e/ou ligamentares, além de encurtamentos musculares e desuso (2).

Não houve associação entre o lado afetado pelo zumbido e o lado da restrição de amplitude de movimento nos indivíduos deste estudo, porém houve uma tendência a tal associação possivelmente pela existência, nos casos 
de tensão muscular, de uma conexão entre os aferentes proprioceptivos e nociceptivos da região cervical e o núcleo coclear (9). Acredita-se a partir da literatura da área e dos dados obtidos neste estudo, que em pesquisas com uma população maior possa ser evidenciada uma associação entre o lado da restrição de amplitude de movimento e o lado do zumbido.

\section{CONCLUSÃO}

Conclui-se que o zumbido e a cervicalgia tem prevalência importante nos idosos e que, com o envelhecimento há uma diminuição na amplitude de movimento cervical em todos os graus - flexão, extensão, rotação cervical e flexão lateral sendo a maior limitação em rotação cervical para a direita.

Não houve associação entre zumbido e cervicalgia nem entre zumbido e diminuição de amplitude de cervical nesta população. Também não ocorreu associação entre o lado afetado pelo zumbido e o lado da restrição de amplitude de movimento, nos indivíduos com queixa de zumbido. Os resultados deste estudo preliminar demonstram a necessidade de mais pesquisas e do avanço no número desta amostra, para realmente se chegar à conclusão destas associações.

\section{REFERÊNCIAS BIBLIOGRÁFICAS}

1. Ganança FF, Gazzola JM, Ganança CF, Caovilla HH, Ganança MM, Cruz OLM. Quedas em idosos com Vertigem Posicional Paroxística Benigna. BrazJOtorhinolaryngol. 2010, 76(1):113-20.

2. Carvalho CO, Magalhães DAS, Silva Junior JAA, Bicalho LFH, Costa APB, Costa LOP, Figueiredo VF. Estudo comparativo das amplitudes de movimento da coluna cervical em idosos com diferentes níveis de aptidão física. Acta Fisiatr. 2006, (13):347-51.

3. Pinto PCL, Sanchez TG, Tomita S. Avaliação da relação entre severidade do zumbido e perda auditiva, sexo e idade do paciente. Braz J Otorhinolaryngol. 2010, 76(1):1824 .

4. Ferreira LMBM, Ramos NA, Mendes EP. Caracterização do zumbido em idosos e de possíveis transtornos relacionados. Rev Bras Otorrinolaringol. 2009, 75(2):245-8.

5. Biensinge A, Reibhauer A, Mazurek B. Halswirbelsäule. The role of the cervical spine and the craniomandibular system in the pathogenesis of tinnitus. Somatosensory tinnitus. HNO. 2008, 56:673-677.
6. Okada DM, Onishi ET, Chami FL, Borin A, Cassola N, Guerreiro VM. O uso da acupuntura para alívio imediato do zumbido. Rev Bras Otorrinolaringol. 2006, 72(2):182-6.

7. Lasisi AO, Abiona T, Gureje O. Tinnitus in the elderly: Profile, correlates and impact in the Nigery study of ageing. Otolaryngol Head Neck Surg. 2010, 143:510-515.

8. Michikawa T, Nishiwaki Y, Kikuchi Y, Saito H, Mizutari K, Okamoto M, and Takebayashi T. Prevalence and Factors Associated with Tinnitus: A Community-Based Study of Japanese Elders. J Epidemiol. 2010, 20(4):271-6.

9. Rocha CACB, Sanchez TG, Siqueira JTT. Pontos-gatilho Miofasciais: Ocorrência e Capacidade de Modulação em Pacientes com Zumbido. Arq Int Otorrinolaringol. 2006, 10(3):210-7.

10. Hamill-Ruth RJ; Ruth RA; Cyd CD, Cook A. Management of Tinnitus and hiperacusis using a multidisciplinary pain model. APS Bulletin. 2000, 10(5):126-7.

11. Spidurso WW. Dimensões físicas do envelhecimento. Barueri: Manole; 2005

12. Kapandji IA. Fisiologia articular: esquemas comentados de mecânica vertebral: tronco e coluna vertebral. $5^{\underline{a}}$ ed. São Paulo: Pan-Americana; 2000.

13. Marques AP. Manual de Goniometria. $2^{\underline{a}}$ ed. São Paulo: Manole; 2003

14. Steinmetz LG, Zeigelboim BS, Lacerda AB, Morata TC, Marques JM. Características do zumbido em trabalhadores expostos a ruídos. Rev Bras Otorrinolaringol. 2009, 75(1):714.

15. Pinto PCL, Hoshino AC, Tomita S. Características dos pacientes com queixa de zumbido atendidos em ambulatório especializado - HUCFF. Cad Saúde Coletiva. 2008, 16(3):437-48.

16. Kettler A, Werner K, and Wilke HJ. Morphological changes of cervical facet joints in elderly individuals. Eur Spine J. 2007, 16(7):987-92.

17. Chaves TC, Nagamine HM, Belli JFC, de Hannai MCT, Bevilaqua-Grossi D, Oliveira AS. Confiabilidade da fleximetria e goniometria na avaliação da amplitude de movimento cervical em crianças. Rev Bras Fisioter. 2008, 12(4): 283-9.

18. Björne, A. Assessment of temporomandibular and cervical spine disorders in tinnitus patients. Prog Brain Res. 2007, 166:216-9. 